\title{
Expression of matrix metalloproteinases in pigs with hyperoxia-induced acute lung injury
}

\author{
Y. Gushima, K. Ichikado, M. Suga, T. Okamoto, K. Iyonaga, K. Sato, H. Miyakawa, M. Ando
}

\begin{abstract}
Expression of matrix metalloproteinases in pigs with hyperoxia-induced acute lung injury. Y. Gushima, K. Ichikado, M. Suga, T. Okamoto, K. Iyonaga, K. Sato, H. Miyakawa, M. Ando. (C) ERS Journals Ltd 2001.

ABSTRACT: The aim of this study was to determine the role of matrix metalloproteinases (MMPs) in the pathogenesis of acute lung injury induced by hyperoxia.

Twenty-three pigs were exposed in sealed cages to $>80 \%$ oxygen (for $24-120$ h) or room air. Correlation between MMP-2/MMP-9 activity, measured by gelatin zymography in bronchoalveolar lavage fluid (BALF), and the histological findings and pathological parameters were examined in detail. Sources of these MMPs in the hyperoxic lung were analysed by immunohistochemistry.

The histological progression of acute lung injury in this model ranged from the early exudative to the early proliferative phase of diffuse alveolar damage (DAD). MMP-2 and $\mathbf{- 9}$ activities were elevated under prolonged hyperoxic exposure. MMP-9 activity correlated significantly with the oxygen tension in arterial blood/inspiratory oxygen fraction, the lung wet-to-dry weight ratio, and the number of neutrophils in BALF, whereas MMP-2 activity did not correlate at all with these factors. MMP-9 activity correlated more closely with the pathological findings of DAD than did MMP-2 activity. Strong MMP-9 expression was observed in neutrophils, alveolar macrophages as well as alveolar lining epithelial cells.

These results suggest that matrix metalloproteinase, which may derive from neutrophils recruited into airspaces, plays an important role in the pathogenesis of hyperoxic diffuse alveolar damage.

Eur Respir J 2001; 18: 827-837.
\end{abstract}

First Dept of Internal Medicine, Kumamoto University School of Medicine, Kumamoto, Japan.

Correspondence: M. Suga, First Dept of Internal Medicine, Kumamoto University School of Medicine, 1-1-1 Honjo, Kumamoto 860-0811, Japan. Fax: 81963710582

Keywords: Acute lung injury
elastase
hyperoxia
matrix metalloproteinases

matrix metalloproteinases

Received: July 182000

Accepted after revision May 282001

This work was supported by a grant-inaid for interstitial lung diseases from the Japanese Ministry of Health and Welfare, and a grant-in-aid for scientific research 12670565 from the Japanese Ministry of Education, Culture, Sports, Science and Technology.
High concentration oxygen $\left(\mathrm{O}_{2}\right)$ has been used widely in patients with respiratory failure to improve hypoxaemia. However, $\mathrm{O}_{2}$ can adversely affect all tissues of the body, especially the lung, because the lung is exposed directly to the highest partial pressure of inspired $\mathrm{O}_{2}$. Lung damage induced by hyperoxia is characterized pathologically by diffuse alveolar damage (DAD) [1, 2]. Hyperoxic lung injury is the most life-threatening problem in the treatment of respiratory failure, and many studies have been carried out on the pathogenesis of acute hyperoxic lung injury.

In the pathogenesis of acute lung injury, degradation of the extracellular matrix is considered one of the crucial events. Various proteolytic enzymes are involved in this condition, such as neutrophil elastase and the matrix metalloproteinases (MMPs) [3]. Neutrophil elastase can degrade elastin, proteoglycan, fibronectin and type III collagen, structural components of lung alveoli [4]. MMP-2 (72 kDa gelatinase, gelatinase A) and MMP-9 (92 kDa gelatinase, gelatinase B) have a capacity to degrade gelatin, elastin, fibronectin and type IV collagen, which are major structural components of the basement membrane $[5,6]$. These MMPs are secreted as latent forms by a variety of cell types and are activated at extracellular spaces by serine proteases and other MMP members [7].

Several studies have investigated the roles of these proteinases in acute lung injury [7-11]. However, the precise contributions of these proteinases to the development of acute hyperoxic lung injury, including the relationship between proteinases and disease severity/ pathological changes, are still unclear.

Hyperoxia-exposed experimental animals are useful in evaluating the pathological process of acute lung injury/DAD, because evolution of the lesion can be studied over a definite time period [1,2]. Many studies focusing on the histopathological features have been done in rodent [7-9, 11] or baboon models [12, 13]. However, rodents are different from humans in many ways, especially with regard to lung anatomy and the behaviour of inflammatory mediators or cytokines. From this point of view, the pig is particularly useful 
as an animal model of acute hyperoxic lung injury, because the pig lung resembles the human lung anatomically and immunologically $[14,15]$. However, to the present authors' knowledge, no study has been undertaken in pigs.

The aim of the present study was to clarify the roles of MMPs in the pathogenesis of hyperoxia-induced acute lung injury in a porcine model.

\section{Material and methods}

The protocol of this experimental study was approved by the ethical committee on animal experiments of the Kumamoto University School of Medicine, Kumathoto, Japan.

\section{Animals}

Twenty-three female Yorkshire pigs between 6-9 weeks old (Shizuoka Laboratory Animal Center, Shizuoka, Japan) and weighing 12-15 kg were used in the study.

\section{Hyperoxia exposure}

Animals were housed in individual stainless-steel cages (Natsume Seisakusho Co., Tokyo, Japan) and exposed to hyperoxia as described previously [16]. The concentration of $\mathrm{O}_{2}$ was maintained at $>80 \%$. Nineteen animals were exposed to hyperoxia for $24 \mathrm{~h}$ $(\mathrm{n}=4), 48 \mathrm{~h}(\mathrm{n}=4), 72 \mathrm{~h}(\mathrm{n}=4), 96 \mathrm{~h}(\mathrm{n}=4)$, and $120 \mathrm{~h}$ $(n=3)$. Four pigs exposed to room air in the same cage served as controls. After exposure to hyperoxia, all animals were anaesthetized and mechanically ventilated. After $20 \mathrm{~min}$, an arterial blood sample was obtained from the left carotid artery, and bronchoalveolar lavage (BAL) was performed as described later. Arterial gas tensions were determined with a blood gas analyser (Bayer Medical Ltd, Tokyo, Japan), and the number of peripheral blood leukocytes was determined with a haemocytometer. At the completion of each experiment, the animals were killed with an overdose of intravenous pentobarbital sodium, and lung tissues were obtained.

\section{Bronchoalveolar lavage}

A bronchoscope was inserted into the lower lobe of the right lung via an endotracheal tube. BAL was performed by instillation of $20 \mathrm{~mL}$ of sterilized saline, and fluid was collected by gentle suction. The BAL fluid (BALF) was centrifuged at $200 \times g$ at $4{ }^{\circ} \mathrm{C}$ for $10 \mathrm{~min}$, and the supernatant was aliquoted and frozen at $-80^{\circ} \mathrm{C}$ until use. The cell numbers were counted by haemocytometry and then prepared for cytocentrifuge by plating on to poly-L-lysine coated slides with a Cytospin III (Shandon, Cheshire, UK). Cell smears were stained with standard May-Grünwald-Giemsa stain, and differential cell counts were determined based on 500 cells.

\section{Lung wet-to-dry weight ratio}

To evaluate the severity of pulmonary oedema, the lung wet-to-dry weight ratio was determined [17]. A part of the lower lobe of the left lung was removed, and its wet weight was determined. Specimens were freeze-dried for 2 days and weighed again to determine the dry weight.

\section{Elastase activity}

Elastase activity in BALF was determined as previously described [18]. Briefly, $100 \mu \mathrm{L}$ of BALF and $400 \mu \mathrm{L}$ of specific synthetic elastase substrate methoxysuccinyl-Ala-Ala-Pro-Val p-nitroanilide (1.25 mM) (Sigma Chemical Co., St. Louis, MO, USA) were incubated on a 96 -well plate at $37^{\circ} \mathrm{C}$ for $18 \mathrm{~h}$. After incubation, the amount of degraded substrate was measured by an increased optical density at $405 \mathrm{~nm}$. A standard curve was constructed using similar determinations with human leukocyte elastase (Sigma), and elastase activity in BALF was expressed $\mathrm{ng} \cdot \mathrm{mL}^{-1}$.

\section{Pathological evaluation}

Each left lung removed was inflated with air, fixed with $10 \%$ formalin injected via the left main bronchus, immersed in fixative for 2 days, and subjected to haematoxylin-eosin, azan, and elastica van Gieson stainings. Structural changes within the left lower lobe were evaluated by two independent observers, who had no knowledge of the duration of hyperoxic exposure. The following 13 pathological findings, all recognized characteristics of DAD, were assessed: epithelial destruction, capillary congestion, interstitial oedema, intra-alveolar oedema, haemorrhage, mononuclear infiltration, polymorphonuclear infiltration, interlobular septal thickening, hyaline membrane formation, microatelectasis, type II pneumocyte hyperplasia, fibroblast proliferation, and interstitial collagenous deposition. Findings were graded on a four-point scale $(0=$ absent, $1=$ mild, $2=$ moderate, $3=$ severe). An overall histological score was calculated by totalling the scores. Pathological findings were classified according to the pathological phases of DAD, based on the following criteria: the exudative phase as characterized by hyaline membrane formation and intra-alveolar oedema; the early proliferative phase as characterized by type II pneumocyte hyperplasia with proliferation of fibroblasts; the late proliferative phase as characterized by type II pneumocyte hyperplasia with proliferation of fibroblasts not only within the interstitium, but also within alveolar spaces; the fibrotic phase as characterized by extensive interstitial fibrosis and collagenous connective tissue deposition $[19,20]$. 


\section{Gelatin zymography}

To detect the gelatinolytic activity in BALF and lung tissue, the samples were analysed by gelatin zymography as described previously [21]. Unfixed frozen lung specimens were homogenized in $10 \mathrm{mM}$ Tris- $\mathrm{HCl}$ (pH 7.6) containing $0.2 \mathrm{mM}$ phenylmethylsulphonyl fluoride (Sigma) and $1 \mu \mathrm{g} \cdot \mathrm{mL}^{-1}$ leupeptin (Sigma) by use of a Polytron homogenizer (Kinematica, Lucerne, Switzerland). Briefly, unconcentrated BALF supernatants $(20 \mu \mathrm{L})$ and the homogenates of $0.4 \mathrm{mg}$ of lung tissue $(20 \mu \mathrm{L})$ were treated under nonreducing conditions and subjected to sodium dodecyl sulphate-polyacrylamide gel electrophoresis (SDS-PAGE) in 10\% acrylamide gels containing $0.8 \mathrm{mg} \cdot \mathrm{mL}^{-1}$ alpha-gelatin monomer (Serva Feinbiochemica GmbH \& Co., Heidelberg, Germany) at a constant current of $25 \mathrm{~mA}$ at $0^{\circ} \mathrm{C}$. After electrophoresis, the gels were washed three times with $40 \mathrm{mM}$ Tris- $\mathrm{HCl}$ (pH 7.6), $10 \mathrm{mM} \mathrm{CaCl}, 2 \mu \mathrm{M}$ $\mathrm{ZnCl}_{2}$, and $0.1 \% 23$ lauryl ether (Brij 35) containing 3\% Triton $\mathrm{X}-100$ for $15 \mathrm{~min}$ to remove SDS, and then incubated for $24 \mathrm{~h}$ at $37^{\circ} \mathrm{C}$ in the same buffer, but without Triton X-100. Following incubation, the gels were fixed in a solution of $10 \%$ acetic acid and 50\% methanol and stained with Coomassie brilliant blue R-250 for $60 \mathrm{~min}$. Gelatin digestion was identified as a clear lytic zone against a blue background. The zymographic gels were converted to digital images, and the density (number of pixels) of the lytic zone was used to measure the gelatinase activity by National Institutes of Health (NIH) Image v.1.61 shareware (Research Services Branch of the National Institute of Mental Health, NIH, MD, USA).

When various concentrations of purified human MMP-9 (isolated from human neutrophils) or MMP-2 (Biogenesis Inc., Sandown, NH, USA) were subjected to gelatin zymography as described previously, the density of the gelatinolytic band in the gel correlated linearly with concentrations of purified human MMP2 and -9 after log transformation [21]. Therefore, the MMP amounts in the obtained BAL fluids or lung tissues could be quantitatively assessed by measuring their respective gelatinolytic activity by gelatin zymography. Activity of each MMP was expressed as an arbitrary unit defined as the activity that produced a lytic density of 1 pixel after gelatinolytic reaction in the gel.

\section{Immunohistochemistry}

Goat polyclonal antibodies against human MMP-9 (Santa Cruz Biotechnology Inc., Santa Cruz, CA, USA) and mouse monoclonal antibodies against human MMP-2 (Fuji Chemical Industries Ltd, Toyama, Japan) were used for immunohistochemical staining. Tissue sections were deparaffinized and rehydrated, and endogenous peroxidase activity was inhibited according to the method reported by Isobe et al. [22]. Sections were stained with the antibodies (diluted 1:200 with phosphate buffered saline containing $0.5 \%$ bovine serum albumin) by the indirect immunoperoxidase method with peroxidaselabelled antigoat (Santa Cruz Biotechnology) or antimouse immunoglobulins [F( $\left.\left.\mathrm{ab}^{\prime}\right) 2\right]$ (Amersham, Little Chalfont, UK).

\section{Data analysis}

Interobserver variability for scoring the severity of changes and pathological phases was quantified as a kappa coefficient of agreement. Statistical analysis was performed by the Mann-Whitney U-test for differences between groups. To quantify the relationship between histological scores and pathological phases, the Mann-Whitney U-test was used. Correlations between histological scores and hyperoxic exposure periods, between histological scores and factors associated with acute lung injury, and between histological scores and gelatinolytic activity of BALF were quantified by Spearman's correlation coefficient. Histological scores, peripheral blood leukocyte counts, BALF cell counts, oxygen tension in arterial blood $\left(\mathrm{Pa}, \mathrm{O}_{2}\right)$ /inspiratory oxygen fraction $\left(\mathrm{FI}, \mathrm{O}_{2}\right)$ ratio, lung wet-to-dry weight ratio, and elastase activities are expressed as mean \pm SEM. A p-value $<0.05$ was considered significant.

\section{Results}

Assessment of acute lung injury after hyperoxic exposure

Several factors associated with acute lung injury changed after hyperoxic exposure (fig. 1). The number of neutrophils in peripheral blood did not differ statistically between hyperoxia-exposed and unexposed pigs (fig. 1a). The $P \mathrm{a}, \mathrm{O}_{2} / F \mathrm{I}, \mathrm{O}_{2}$ ratio decreased significantly with exposure over $72 \mathrm{~h}$ in comparison to that of unexposed pigs (fig. 1b). BALF differential cell analysis showed the number of neutrophils to be significantly elevated after $\mathrm{O}_{2}$ exposure over $72 \mathrm{~h}$ (fig. 1c). The lung wet-to-dry weight ratio increased significantly at $72 \mathrm{~h}$ (fig. 1d), and slightly decreased thereafter.

\section{Pathological findings}

The results of pathological evaluations are shown in figure 2. As described in a previous study [16], pathological features from the exudative phase to the early proliferative phase of DAD were observed in the present study. Light micrographs of lung tissue after $48 \mathrm{~h}$ exposure (fig. 2b) showed mild infiltration of inflammatory cells into interstitium as well as capillary congestion. After $72 \mathrm{~h}$ exposure, hyaline membrane formation, interlobular septal thickening, type II pneumocyte hyperplasia, haemorrhage, and infiltration of neutrophils into alveolar spaces were observed (fig. 2c). After $96 \mathrm{~h}$ exposure, these changes became more severe, and fibroblast proliferation was also noted (fig. 2d). Analysis of azan staining showed that 
a)

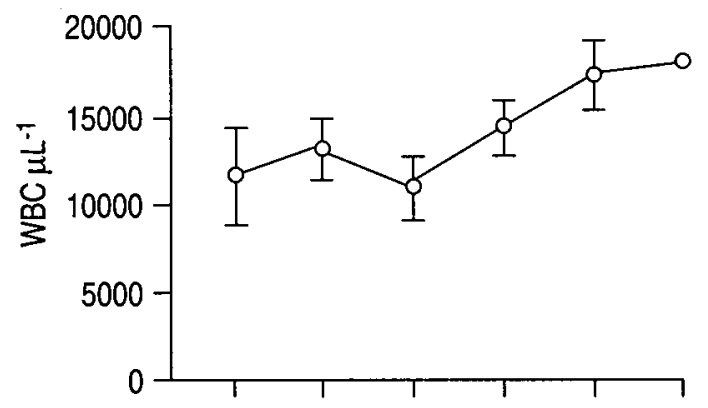

c)

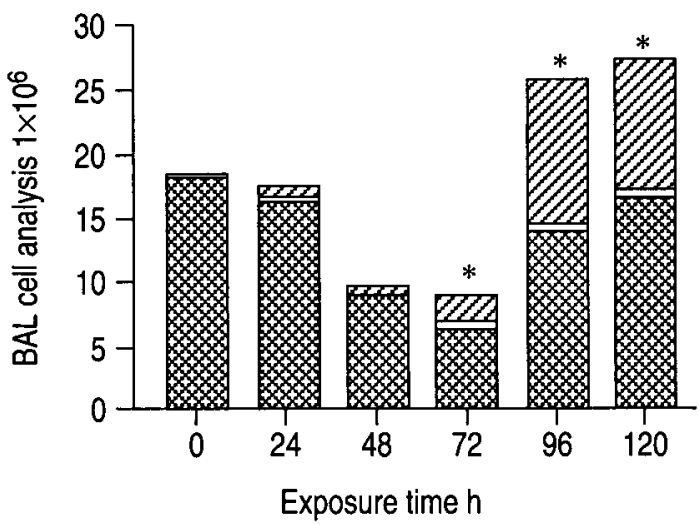

b)

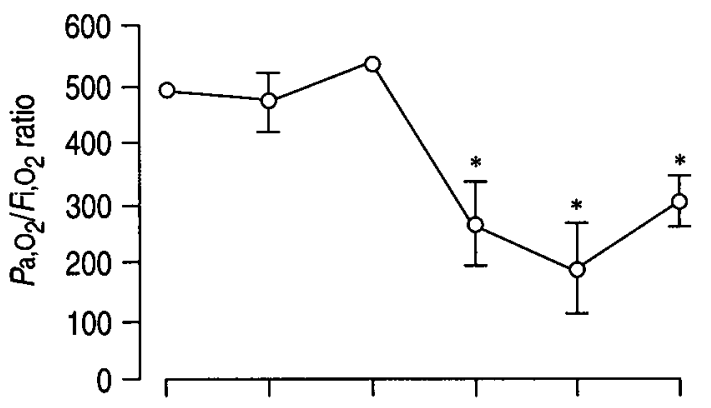

d)

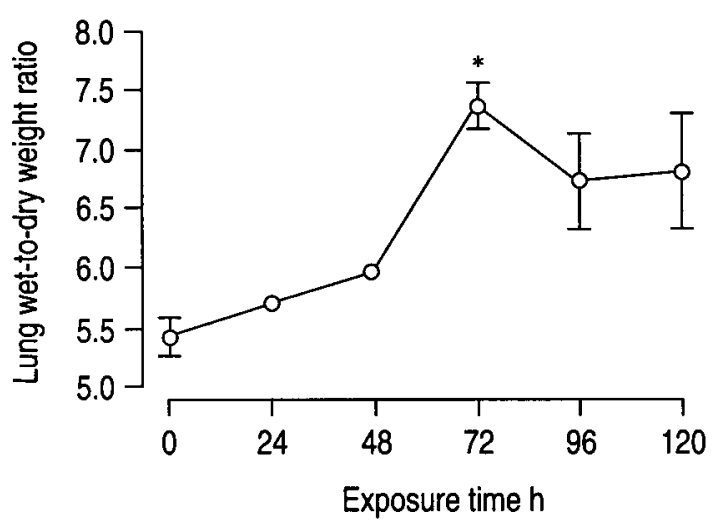

Fig. 1.-Changes in factors associated with acute lung injury after hyperoxic exposure. a) Neutrophil cell counts in peripheral blood do not differ statistically after hyperoxic exposure compared with counts at time zero. b) Oxygen tension in arterial blood $\left(P\right.$ a, $\left.\mathrm{O}_{2}\right) /$ inspiratory oxygen fraction $\left(F \mathrm{I}, \mathrm{O}_{2}\right)$ ratio is significantly decreased in pigs at $>72 \mathrm{~h}$ exposure compared with that of time zero pigs. c) Total cell count, alveolar macrophage cell counts (雄), and lymphocyte cell counts $(\square)$ do not differ statistically compared with counts at time zero, but neutrophil cell counts $(\mathbb{Z})$ are significantly elevated at $>72 \mathrm{~h}$ exposure. d) Lung wet-to-dry weight ratio is significantly increased after $72 \mathrm{~h}$ exposure. BAL: bronchoalveolar lavage; WBC: white blood cell count. Data are presented as mean \pm SEM. $*:$ p $<0.05$ compared to time zero.
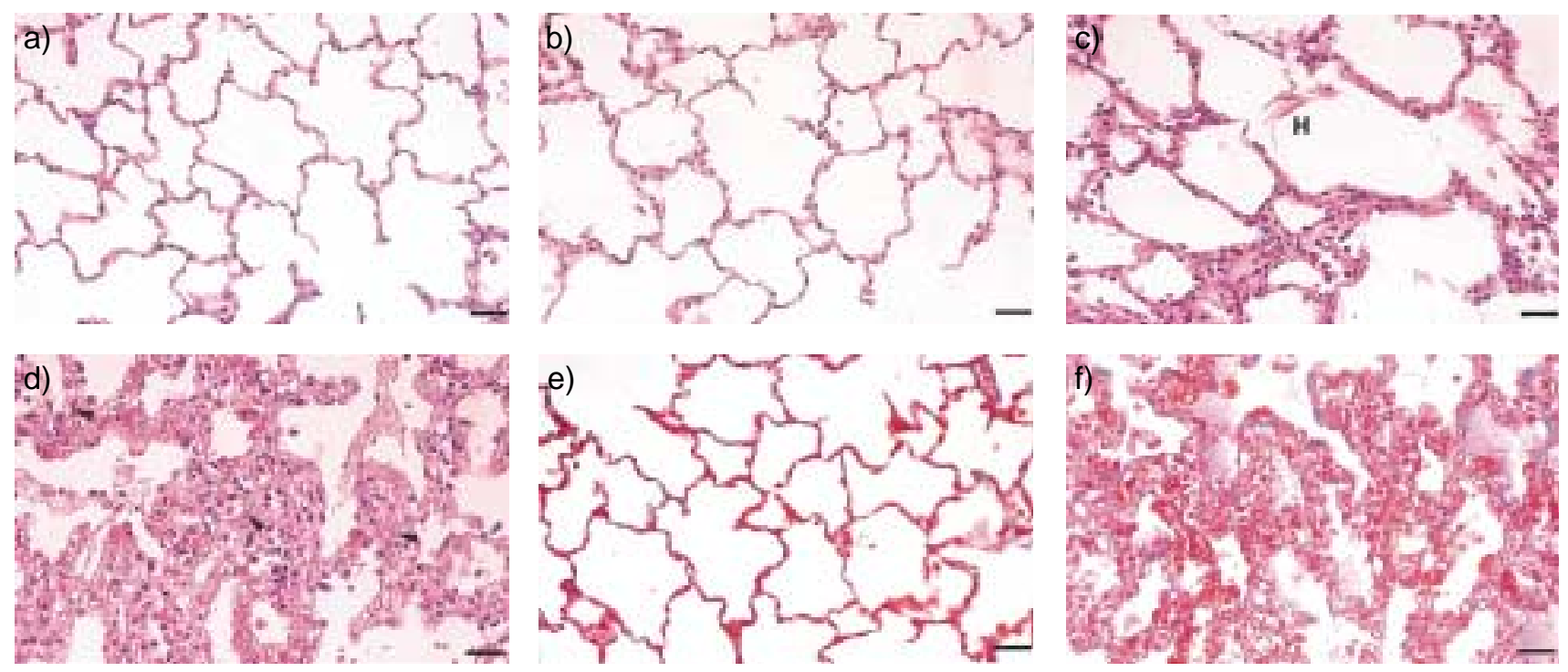

Fig. 2.-Microscopic changes in lungs after hyperoxic exposure. a) No exposure. b) After 48 h exposure, mild infiltration of inflammatory cells into the interstitium is observed. c) After $72 \mathrm{~h}$ exposure, hyaline membrane formation $(\mathrm{H})$, interlobular septal thickening, type II pneumocyte hyperplasia, haemorrhage, and infiltration of neutrophils into alveolar spaces are observed. d) After $96 \mathrm{~h}$ exposure, the findings observed at $72 \mathrm{~h}$ exposure have become severe, and in addition, fibroblast proliferation (arrowheads) is noted. e) No exposure. f) Interstitial collagen deposition did not increase after $96 \mathrm{~h}$ exposure. (Haematoxylin-eosin staining: a-d; azan staining: e and f. Internal scale bars $=50 \mu \mathrm{m})$. 


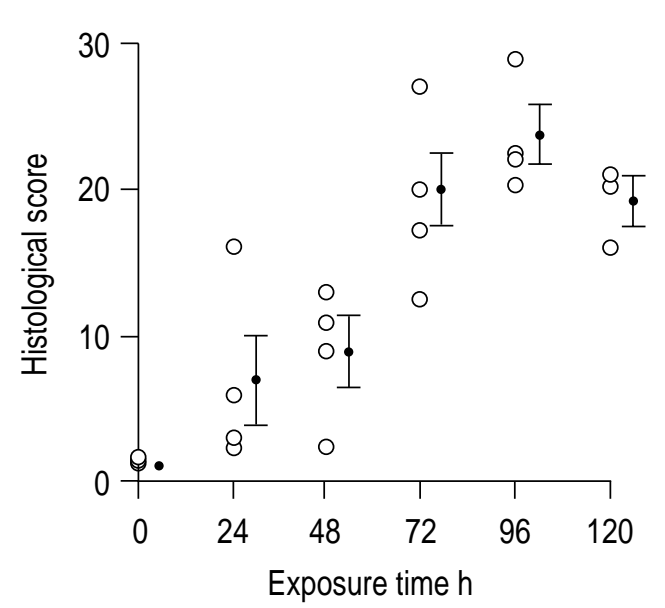

Fig. 3.-Correlation between exposure periods and histological scores. $\bigcirc$ : histological scores for all pigs; $\bigcirc$ : error bars: mean \pm SEM values for every exposure time period. The histological scores elevated time dependently and there was significant correlation between the exposure periods and histological scores (Spearman's correlation coefficient, $\mathrm{r}_{\mathrm{s}}=0.86, \mathrm{p}<0.0001$ ).

interstitial collagenous deposition did not increase after hyperoxic exposure (figs. 2e and f). There was substantial agreement between observers in terms of pathological findings (kappa statistic 0.85). The histological scores that reflect the severity and progression of lung injury elevated time dependently. Moreover, there was significant correlation between the exposure periods and histological scores (Spearman's correlation coefficient, $r_{s}=0.86, p<0.0001$ ) (fig. 3).

\section{Matrix metalloproteinase levels in bronchoalveolar lavage fluid}

Gelatin zymography of pig BALF (fig. 4a) revealed two major gelatinolytic bands of the molecular weight $72 \mathrm{kDa}$ and $92 \mathrm{kDa}$. These two bands showed the same mobility with those of purified human MMP-2 and -9 (lane S), the upper and lower band corresponding to MMP-9 and MMP-2, respectively. Gelatinolytic activities of both MMPs in BALF were quantified using NIH Image (figs. $4 \mathrm{~b}$ and 4c). BALF MMP-2 activities significantly increased at $72 \mathrm{~h}$ and $96 \mathrm{~h}$ and fell to the time-zero level at $120 \mathrm{~h}$ (fig. 4b). MMP-9 expression did not change with exposure $<48 \mathrm{~h}$, but significantly increased with exposure $>72 \mathrm{~h}$, peaked at $96 \mathrm{~h}$, and slightly decreased at $120 \mathrm{~h}$ (fig. 4c). The active forms of MMP-9 (88 kDa) and MMP-2 $(68 \mathrm{kDa})$ were also observed especially in $72 \mathrm{~h}$ and $96 \mathrm{~h}$ exposure. Gelatin zymography of lung homogenate revealed two major gelatinolytic bands corresponding to MMP-9 and MMP-2 (fig. 4e), however their activities did not differ statistically between hyperoxia-exposed and unexposed pigs.

\section{Neutrophil elastase activity}

BALF elastase activity accumulated during hyperoxia in a time-dependent manner, and the increase was significant after exposure over $48 \mathrm{~h}$, in comparison to unexposed pigs (fig. 4d).

\section{Immunohistochemistry}

Weak expression of MMP-2 was detected in alveolar macrophages, alveolar-lining epithelium, alveolar septal interstitium, and interstitial cells in the lungs of unexposed pigs (fig. 5a) and of pigs exposed for $48 \mathrm{~h}$ (fig. 5b). MMP-2 expression in alveolar macrophages increased markedly in pigs exposed to hyperoxia for $96 \mathrm{~h}$ compared to that in unexposed pigs (fig. 5c).

MMP-9 expression was detected in alveolar epithelial cells in unexposed pigs (fig. 5d). No significant immunohistochemical change was observed in pigs exposed for $48 \mathrm{~h}$ compared to that in unexposed pigs (fig. 5e). After $96 \mathrm{~h}$ exposure, strong expression of MMP-9 was observed in alveolar macrophages, neutrophils, and alveolar-lining epithelium (fig. 5f).

\section{Relationship between proteinases and factors of acute lung injury}

The gelatinolytic activities in BALF were correlated with various factors associated with acute lung injury, and the results are shown in figure 6. MMP-9 activity showed a significant negative correlation with the $P a, \mathrm{O}_{2} / F \mathrm{I}, \mathrm{O}_{2}$ ratio (Spearman's correlation coefficient, $\mathrm{r}_{\mathrm{s}}=-0.71, \mathrm{p}=0.0001$ ) (fig. 6b) and significantly correlated with the lung wet-to-dry weight ratio $\left(\mathrm{r}_{\mathrm{s}}=0.52\right.$, $\mathrm{p}=0.01$ ) (fig. 6e). In contrast, MMP-2 activity did not significantly correlate with these parameters (figs. 6a and 6d). The relationship between MMPs and the number of neutrophils in BALF is shown in figures $6 \mathrm{~g}$ and $6 \mathrm{~h}$. MMP-9 activity in BALF significantly correlated with the number of neutrophils $\left(\mathrm{r}_{\mathrm{s}}=0.73, \mathrm{p}<0.0001\right)$ (fig. 6h), but MMP-2 activity did not. Neither MMP-2 nor MMP-9 expression correlated with the number of total cells, alveolar macrophages or lymphocytes (data not shown).

There was a significant correlation between elastase activity in $\mathrm{BALF}$ and $\mathrm{Pa}, \mathrm{O}_{2} / F \mathrm{I}, \mathrm{O}_{2}$ ratio $\left(\mathrm{r}_{\mathrm{s}}=\right.$ -0.43, p<0.05) (fig. 6c), between elastase activity and lung wet-to-dry weight ratio $\left(\mathrm{r}_{\mathrm{s}}=0.63, \mathrm{p}<0.005\right)$ (fig. 6f), and between elastase activity and the number of neutrophils in BALF $\left(r_{s}=0.61, p<0.005\right)$ (fig. 6i).

\section{Relationship between proteinases and histological scores}

The relationship between proteinases in BALF and overall histological scores is shown in figure 7. Activity of both MMP-2 (fig. 7a) and MMP-9 (fig. 7b) significantly correlated with histological scores, although MMP-9 activity had a stronger correlation than did MMP-2 activity (MMP-2, $\mathrm{r}_{\mathrm{s}}=0.51, \mathrm{p}=0.01$; MMP-9, $\left.r_{s}=0.72, p=0.0001\right)$. As with MMP-9, elastase 

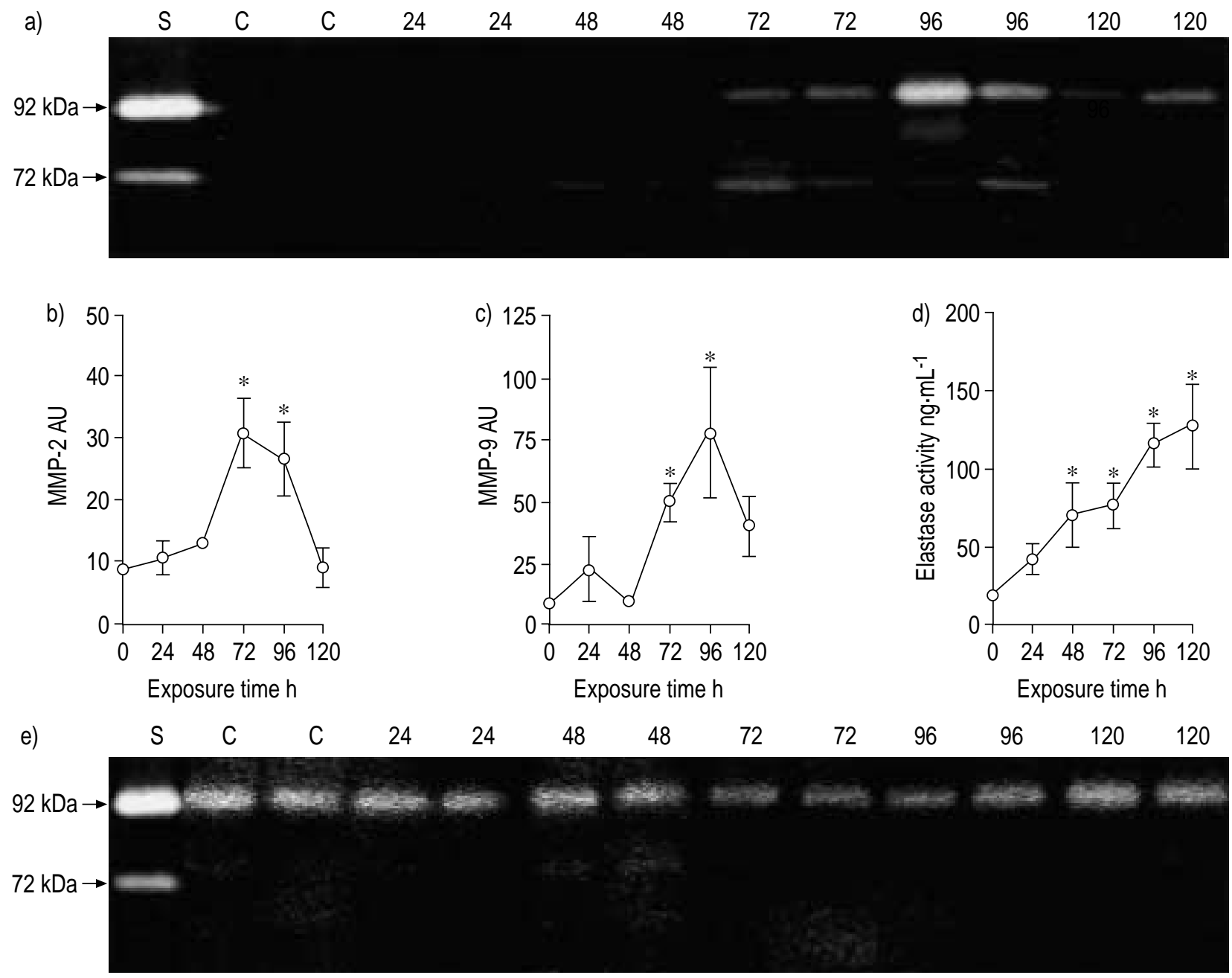

Fig. 4.-Gelatinolytic activities and elastase activities in bronchoalveolar lavage fluid (BALF) from hyperoxia-exposed and unexposed pigs. a) Gelatin zymography in BALF from representative hyperoxia-exposed pigs and from unexposed pigs. Lanes S: purified human matrix metalloproteinase (MMP)-9 and MMP-2; C: BALF from unexposed pigs; 24: BALF from pigs after 24 h exposure; 48 : 48 h; 72 : $72 \mathrm{~h} ; 96: 96 \mathrm{~h} ; 120: 120 \mathrm{~h}$. Gelatinolytic clear bands of the latent form of MMP-9, active form of MMP-9, latent form of MMP-2, and active form of MMP-2 were detected. These bands are clearly visible in BALF from hyperoxia-exposed pigs. b) Densitometric analysis of gelatinolytic bands in BALF corresponding to the latent form of MMP-2. c) Densitometric analysis of gelatinolytic bands in BALF corresponding to the latent form of MMP-9. d) Elastase activities in BALF. e) Gelatin zymography in lung homogenate from representative hyperoxia-exposed pigs and from unexposed pigs. Lanes S: purified human MMP-9 and MMP-2; C: homogenate from unexposed pigs; 24: homogenate from pigs after $24 \mathrm{~h}$ exposure; $48: 48 \mathrm{~h} ; 72: 72 \mathrm{~h} ; 96: 96 \mathrm{~h} ; 120$ : $120 \mathrm{~h}$. Gelatinolytic clear bands of the latent form of MMP-9, and latent form of MMP-2 were detected. These bands do not differ between hyperoxia-exposed and unexposed pigs. AU: arbitrary unit. *: $\mathrm{p}<0.05$ compared to time zero.

activity correlated well with overall histological scores $\left(\mathrm{r}_{\mathrm{s}}=0.76, \mathrm{p}=0.0001\right)$ (fig. $\left.7 \mathrm{c}\right)$. The correlations between these proteinases and the pathological features of DAD are summarized in table 1. MMP-9 and elastase significantly correlated with all factors except interstitial collagen deposition, whereas MMP-2 expression had a significant correlation with eight out of 10 factors characterizing the exudative phase of DAD. With regard to these eight factors, MMP-9 and elastase had stronger correlation than did MMP-2. MMP-2 expression did not have a significant correlation with type II pneumocyte hyperplasia or fibroblast proliferation, which are the hallmarks of the proliferative phase of DAD.

\section{Discussion}

Hyperoxia is one of the major causes of DAD $[1,2]$ and the matter of toxicity is clinically of great importance because it is not possible to carry out a long-term and high-concentration oxygen therapy in patients with severe respiratory failure. Thus, the development of an experimental animal model is very important as it allows the adverse effects of hyperoxia in the lung to be evaluated. For this purpose, a hyperoxic lung injury model was developed in pigs, because the pig lung resembles the human lung not only structurally [14] but also in immunological responses [15]. Recently, the present authors reported 

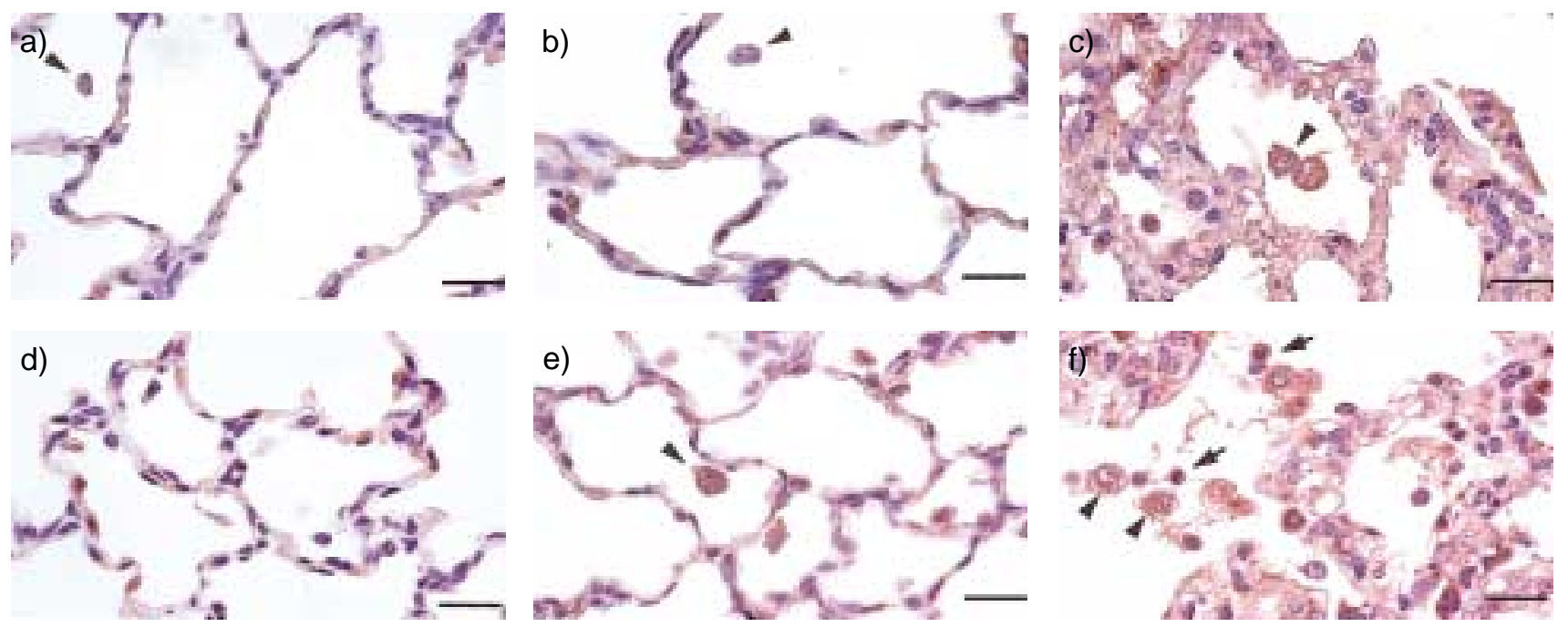

Fig. 5.-Immunohistochemical detection of matrix metalloproteinase (MMP)-2 and MMP-9 in lung tissue. a) Weak MMP-2-expression was observed in alveolar macrophages, alveolar lining epithelium, alveolar septal interstitium, and interstitial cells in unexposed pigs. b) Obvious increase of MMP-2-expression was not detected immunohistochemically in pigs exposed for $48 \mathrm{~h}$. c) After $96 \mathrm{~h}$ exposure, MMP2 was increasingly expressed in alveolar macrophages. d) Weak expression of MMP-9 was detected in alveolar type II cells in unexposed pigs. e) Obvious increase of MMP-9-expression was not detected immunohistochemically in $48 \mathrm{~h}$ exposed pigs. f) After $96 \mathrm{~h}$ exposure, MMP-9 was strongly expressed in alveolar macrophages, neutrophils, and alveolar lining epithelium. Arrowheads: alveolar macrophages; arrows: neutrophils. (Internal scale bars $=30 \mu \mathrm{m}$ ).

that thin-section computed tomography findings of this pig model were very similar to those of human acute respiratory distress syndrome (ARDS) [16], indicating that this model closely resembles human hyperoxic lung injury. However, there are some limitations with this model, as relatively few specific materials, such as enzyme-linked immunosorbent assay systems or antibodies, are commercially available. Moreover, protein or messenger ribonucleic acid (mRNA) sequences are not available on database systems. Therefore, enzyme activities of MMP and neutrophil elastase were measured and compared with lung injury parameters or histological findings.

In this pig model, various lung injury parameters such as $\mathrm{Pa}, \mathrm{O}_{2} / \mathrm{FI}, \mathrm{O}_{2}$ ratio, lung wet-to-dry weight ratio, and number of neutrophils in BALF, all deteriorated by hyperoxic exposure time dependently, particularly with exposure for $>72 \mathrm{~h}$ (fig. 1). Moreover, the histological scores (table 1) which reflect the severity and progression of lung injury, also elevated time dependently and strongly correlated with exposure time (fig. 3). The model presented in this study closely resembled human DAD, particularly from the exudative to the early proliferative phase. Moreover, this histological scoring system could be useful in quantitative evaluation of pathological changes, not only in animal DAD model but also in human acute lung injury.

In the mammalian lung, the extracellular matrix is very important in the maintenance of both structural and functional integrity. The extracellular matrix consists of collagenous protein such as collagen types I, III, and IV and noncollagenous protein such as elastin, fibronectin, laminin and proteoglycan [23]. Among these, type IV collagen is the major component of the basement membrane. Both epithelial and endothelial basement membrane destruction are important in the pathogenesis of idiopathic pulmonary fibrosis (IPF) [24] or ARDS [25]. Thus, the present study focused on the type IV collagenolytic MMPs such as MMP-2 and MMP-9. Several investigators have reported the involvement of MMPs in lung injury in humans and animal models. Increased activities of MMP-2 and MMP-9 in BALF are reported in patients with IPF [21] and ARDS [10]. In addition, increased BALF MMP levels were also reported in a rat model of hyperoxia [9] and after intratracheal instillation of lipopolysaccharide in guinea pigs [8]. However, there has been no study assessing the expression of these proteinases from the exudative to the early proliferative phase of DAD, or analysing the relationship between MMP expression and specific pathological parameters.

In immunohistochemical evaluation, neutrophils, alveolar macrophages, and alveolar-lining epithelial cells showed strong MMP-9 expression (fig. 5). However, MMP-9 activity in BALF correlated only with the number of neutrophils, suggesting that these cells are among the major sources of MMP-9 [26]. Hyperoxia is known to stimulate alveolar macrophages and epithelial cells to produce chemokines such as interleukin-8 into the alveolar space, which attract the neutrophils into alveolar space. In this process, neutrophils release huge amounts of MMP-9 from their stored granules, which are thought to assist in their migration through the endothelial or epithelial basement membranes, and may destroy the pulmonary parenchyma resulting in lung injury.

In general, 10-20 kDa of the total molecular weight of the pro-MMPs is lost during their activation, due to proteolytic removal of their propeptide domain 

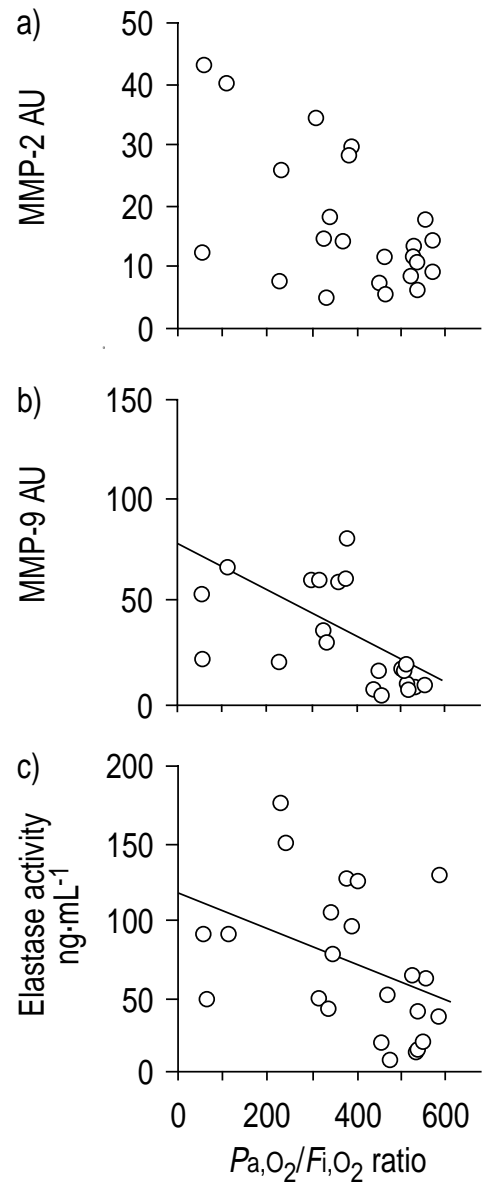

d)

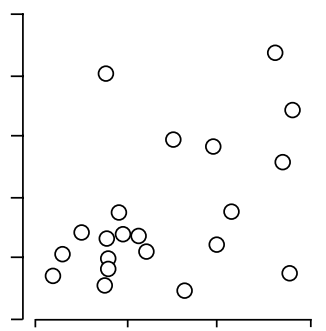

e)

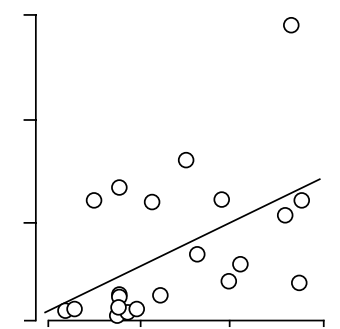

f)

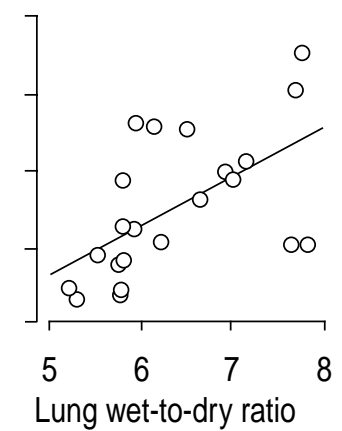

g)

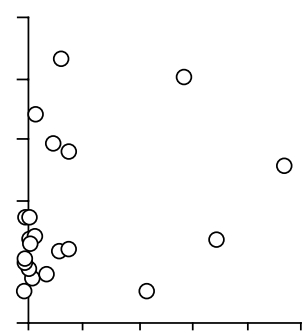

h)

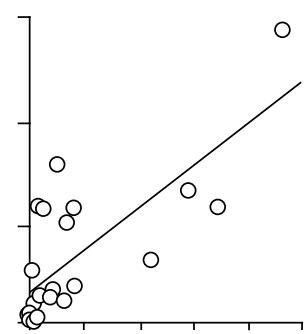

i)

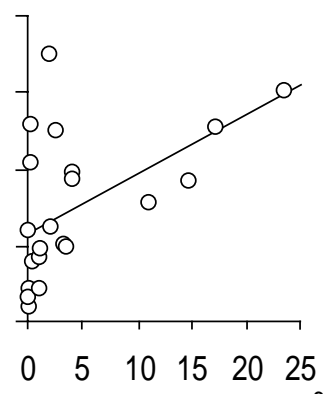

Fig. 6. - Correlation between proteinases in bronchoalveolar lavage fluid (BALF) and factors associated with acute lung injury. Note that matrix metalloproteinase (MMP)-2 expression is not correlated with the oxygen tension in arterial blood $\left(P \mathrm{a}, \mathrm{O}_{2}\right) /$ inspiratory oxygen fraction $\left(F \mathrm{I}, \mathrm{O}_{2}\right)$ ratio $\left(\mathrm{r}_{\mathrm{s}}=-0.40, \mathrm{p}=0.06\right)$ (a), lung wet-to-dry weight ratio $\left(\mathrm{r}_{\mathrm{s}}=0.40, \mathrm{p}=0.06\right)(\mathrm{d})$, or number of neutrophils in $\mathrm{BALF}\left(\mathrm{r}_{\mathrm{s}}=0.33\right.$, $\mathrm{p}=0.12)(\mathrm{g})$; whereas, MMP-9 expression has a clear negative correlation with the $P \mathrm{a}_{\mathrm{a}} \mathrm{O}_{2} / F_{\mathrm{I}}, \mathrm{O}_{2} \quad$ ratio $\left(\mathrm{r}_{\mathrm{s}}=-0.71\right.$, $\left.\mathrm{p}=0.0001\right)(\mathrm{b})$ and is significantly correlated with the lung wet-to-dry weight ratio $\left(\mathrm{r}_{\mathrm{s}}=0.52, \mathrm{p}=0.01\right)$ (e) and number of neutrophils in $\mathrm{BALF}\left(\mathrm{r}_{\mathrm{s}}=0.73\right.$, $\left.\mathrm{p}<0.0001\right)$ (h). As with MMP-9, elastase activity in BALF correlates with $P_{\mathrm{a}}, \mathrm{O}_{2} / F_{\mathrm{I}}, \mathrm{O}_{2}$ ratio $\left(\mathrm{r}_{\mathrm{s}}=-0.43\right.$, p $\left.<0.05\right)$ (c), with lung wet-to-dry weight ratio $\left(r_{s}=0.63, p<0.005\right)(f)$, and with number of neutrophils in BALF $\left(r_{s}=0.61, p<0.005\right)$ (i). AU: arbitrary unit.

[27]. Most of the MMP-9 secreted into BALF in the present model was in a $92 \mathrm{kDa}$ pro-form, whereas only small amounts of the $88 \mathrm{kDa}$ active MMP-9 was seen after 72-96 h hyperoxia (fig. 4). The present authors previously reported similar results, showing that the majority of MMP-9 in BALF of IPF patients was in the $92 \mathrm{kDa}$ pro-form [21]. Comparable findings were reported in an immune complex-mediated lung injury model, in which the active forms of MMP-9 were not detected in undiluted BALF unless the samples were concentrated 50-fold [11]. However, the $92 \mathrm{kDa}$ form of MMP-9 may have the potential to degrade extracellular matrix components at the site of injury, since MMPs can also be activated by thiol-modifying chemicals, such as reactive oxygen or nitrogen species $\left(\mathrm{OCl}^{-}, \mathrm{OH}\right.$ radical or peroxynitrite), oxidized glutathione, and organomercurial compounds, through the chemical modification of the cysteine residue in the pro-peptide domain. In this case, proteolytic cleavage of the MMP pro-peptide is not required [28]. The authors speculate that reactive oxygen or nitrogen species may participate in the activation of MMPs at the site of lung injury. Another possibility is that active forms detected by zymography may not reflect all the active forms present in the lung. Additionally, active MMP may interact with lung extracellular matrix protein and may therefore not appear in BALF [29, 30].

MMP-2 expression in BALF tended to elevate much earlier than MMP-9 (fig. 4), and was localized mainly in alveolar macrophages and alveolar lining epithelia, which are initially exposed to the high $\mathrm{O}_{2}$ levels in this model (fig. 5). MMP-2 may be produced by alveolar macrophages and alveolar lining epithelia in response to hyperoxic conditions and may lead to the earliest pathological changes of DAD before obvious lung injury occurs. The function of this "early-elevating" MMP-2 is not clear, but the finding that MMP-2 activity correlated less strongly with pathological features of DAD than MMP-9 (table 1) indicates that MMP-2 contributed relatively little to the observed lung injury. 

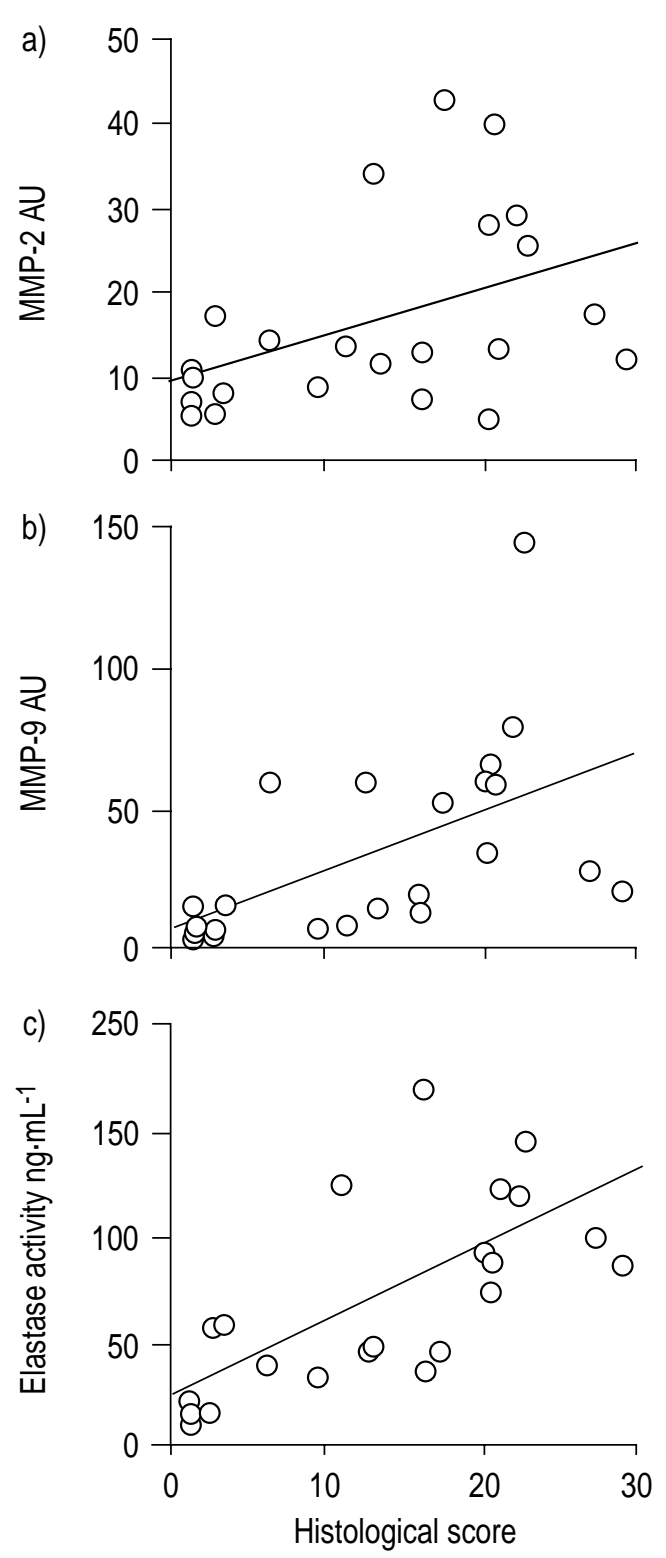

Fig. 7.-Correlation between proteinases in bronchoalveolar lavage fluid and overall histological scores. Note that expression of both matrix metalloproteinase (MMP)-2 (a) and MMP-9 (b) is significantly correlated with histological scores, but MMP-9 expression shows a stronger correlation than does MMP-2 (Spearman's correlation coefficient for MMP-2: $r_{s}=0.51, p=0.01$, MMP-9: $r_{s}=0.72$, $\mathrm{p}=0.0001)$. The correlation between elastase and overall histological scores is similar to that of MMP-9 $\left(\mathrm{r}_{\mathrm{s}}=0.76, \mathrm{p}=0.0001\right)$ (c) AU: arbitrary units.

Both MMP-2 and MMP-9 expression were detected in all samples of homogenated lung tissue; however, the levels of expression did not differ between hyperoxia-exposed and unexposed pigs. Although the precise reasons for the different results obtained from the homogenate of lung and BALF zymograms are unclear, there are several possibilities. Firstly, the frozen lung tissues contained large numbers of blood cells such as neutrophils and monocytes/ macrophages because the tissues were not perfused with saline. Thus, lung homogenates contained blood cell-derived MMPs, which would eliminate the difference of parenchymal expression of MMPs between the hyperoxia- and normoxia-exposed lung tissues. Secondly, the lung tissues probably consisted of various types of cells such as bronchiolar epithelial cells, alveolar epithelial cells, fibroblasts, and other interstitial cells. All of these cells potentially express MMPs, so the zymographic data from the lung homogenates reflect the total MMP activity from the various lung cells, diminishing the differences between the hyperoxia- and normoxia-exposed lung tissues. Thirdly, most cells (except some inflammatory cells such as neutrophils, eosinophils and mast cells) are reported to produce MMPs through constitutive pathways. They secrete MMPs to the outside of the cells immediately after synthesis. Accordingly, MMP activity in the BALF reflects MMPs secreted to the outside of the cells; thus, the authors believe that the BALF zymogram can clarify pathological states much better than the zymogram of tissue homogenates.

The present study also found that neutrophil elastase levels in BALF correlated significantly with factors associated with lung injury and pathological findings (fig. 6). The activity of neutrophil elastase is regulated by alpha-1 protease inhibitor, which can be degraded by several MMPs (including MMP-7, -8 and -9), thus creating a protease-antiprotease imbalance in neutrophil elastase activity. On the other hand, neutrophil elastase is able to activate proMMPs through the proteolytic removal of their prodomain. Thus, neutrophil elastase and MMPs may synergistically increase each other's activities and play important roles in the pathogenesis and severity of DAD.

In summary, the authors demonstrated that the pathological changes in this acute hyperoxic lung injury model range from the exudative to the early proliferative phase of diffuse alveolar damage and that matrix metalloproteinase-2, matrix metalloproteinase -9 , and elastase increase with disease progression in hyperoxia-exposed lungs. Matrix metalloproteinase-9 and elastase activity in bronchoalveolar lavage fluid correlated well with factors associated with acute lung injury and with characteristic, pathological changes. In contrast, matrix metalloproteinase-2 activity in bronchoalveolar lavage fluid did not correlate with these factors, but showed a weak correlation with some histological parameters. The importance of these proteinases in hyperoxia is likely to be elucidated more clearly in future studies, with pharmacological inhibitors of these enzymes, or in studies with transgenic animals. Such studies will determine whether matrix metalloproteinase inhibitors, in particular, may become practical for suppressing disease development in acute respiratory distress syndrome/diffuse alveolar damage.

\footnotetext{
Acknowledgements. The authors sincerely thank A. van der Vliet for critical reading of the manuscript and helpful discussions.
} 
Table 1. - Relationship between matrix metalloproteinases (MMPs)/elastase and pathological features of diffuse alveolar damage

\begin{tabular}{|c|c|c|c|c|c|c|}
\hline & \multicolumn{2}{|c|}{ MMP-2 } & \multicolumn{2}{|c|}{ MMP-9 } & \multicolumn{2}{|c|}{ Elastase } \\
\hline & $\mathrm{r}_{\mathrm{s}}$ & p-value & $\mathrm{r}_{\mathrm{s}}$ & p-value & $\mathrm{r}_{\mathrm{s}}$ & $\mathrm{p}$-value \\
\hline \multicolumn{7}{|l|}{ Exudative phase } \\
\hline Epithelial destruction & $0.51 *$ & 0.013 & $0.64^{\#}$ & 0.001 & $0.64^{\#}$ & 0.001 \\
\hline Capillary congestion & $0.60^{\#}$ & 0.003 & $0.80^{\#}$ & $<0.0001$ & $0.69^{\#}$ & 0.0003 \\
\hline Interstitial oedema & $0.55^{* *}$ & 0.007 & $0.66^{\#}$ & 0.001 & $0.67^{\#}$ & 0.0004 \\
\hline Alveolar oedema & $0.45^{*}$ & 0.030 & $0.56^{* *}$ & 0.005 & $0.65^{\#}$ & 0.0008 \\
\hline Haemorrhage & 0.40 & NS & $0.53^{*}$ & 0.010 & $0.70^{\#}$ & 0.0002 \\
\hline Mononuclear infiltration & 0.37 & NS & $0.65^{\#}$ & 0.001 & $0.63^{\#}$ & 0.001 \\
\hline Polymorphonuclear infiltration & $0.46^{*}$ & 0.026 & $0.57^{\#}$ & 0.005 & $0.66^{\#}$ & 0.0006 \\
\hline Interlobular septal thickening & $0.44 *$ & 0.035 & $0.62^{\#}$ & 0.002 & $0.55^{* *}$ & 0.006 \\
\hline Hyaline membrane & $0.44^{*}$ & 0.034 & $0.71^{\#}$ & 0.0001 & $0.77^{\#}$ & $<0.0001$ \\
\hline Microatelectasis & $0.45^{*}$ & 0.032 & $0.66^{\#}$ & 0.0005 & $0.69^{\#}$ & 0.0003 \\
\hline \multicolumn{7}{|l|}{ Proliferative phase } \\
\hline Type II pneumocyte hyperplasia & 0.41 & NS & $0.58^{\#}$ & 0.004 & $0.66^{\#}$ & 0.0007 \\
\hline Fibroblast proliferation & 0.27 & NS & $0.57^{\#}$ & 0.005 & $0.62^{\#}$ & 0.002 \\
\hline \multicolumn{7}{|l|}{ Fibrotic phase } \\
\hline Interstitial collagen deposition & ND & & ND & & ND & \\
\hline
\end{tabular}

$\mathrm{r}_{\mathrm{s}}$ : Spearmen's correlation coefficient; NS: nonsignificant; ND: not detected. *: $\mathrm{p}<0.05$ compared with MMP-2 or -9 ; **: $\mathrm{p}<0.01$ compared with MMP-2 or -9 ; ${ }^{\#}: \mathrm{p}<0.005$ compared with MMP-2 or -9 .

\section{References}

1. Katzenstein ALA, Bloor CM, Liebow AA. Diffuse alveolar damage - the role of oxygen, shock, and related factors. A review. Am J Pathol 1976; 85: 210-228.

2. Deneke SM, Fanburg BL. Normobaric oxygen toxicity of the lung. N Engl J Med 1980; 303: 76-86.

3. Palmgren MS, deShazo RD, Carter RM, Zimny ML, Shah SV. Mechanisms of neutrophil damage to human alveolar extracellular matrix: the role of serine and metalloproteinases. J Allergy Clin Immunol 1992; 89: 905-915.

4. Campbell EJ, Senior RM, Welgus HG. Extracellular matrix injury during lung inflammation. Chest 1987; 92: 161-167.

5. Woessner JF Jr. Matrix metalloproteinases and their inhibitors in connective tissue remodeling. FASEB $J$ 1991; 5: 2145-2154.

6. Matrisian LM. The matrix-degrading metalloproteinases. Bio Essays 1992; 14: 455-462.

7. Ferry G, Lonchampt M, Pennel L, de Nanteuil G, Canet E, Tucker GC. Activation of MMP-9 by neutrophil elastase in an in vivo model of acute lung injury. FEBS Lett 1997; 402: 111-115.

8. D'Ortho M-P, Jarreau P-H, Delacourt C, et al. Matrix metalloproteinase and elastase activities in LPS-induced acute lung injury in guinea pigs. $\mathrm{Am}$ J Physiol 1994; 266: L209-L216.

9. Pardo A, Selman M, Ridge K, Barrios R, Sznajder JI. Increased expression of gelatinases and collagenase in rat lungs exposed to $100 \%$ oxygen. Am J Respir Crit Care Med 1996; 154: 1067-1075.

10. Torii K, Iida K, Miyazaki Y, et al. Higher concentrations of matrix metalloproteinases in bronchoalveolar lavage fluid of patients with adult respiratory distress syndrome. Am J Respir Crit Care Med 1997; 155: 43-46.

11. Gibbs DF, Shanley TP, Warner RL, Murphy HS, Varani J, Johnson KJ. Role of matrix metalloprotei- nases in models of macrophage-dependent acute lung injury. Evidence for alveolar macrophage as source of proteinases. Am J Respir Cell Mol Biol 1999; 20: 11451154.

12. Johanson WG Jr, Holcomb JR, Coalson JJ. Experimental diffuse alveolar damage in baboons. Am Rev Respir Dis 1982; 126: 142-151.

13. Los Santos RD, Seidenfeld JJ, Anuzueto A, et al. One hundred percent oxygen lung injury in adult baboons. Am Rev Respir Dis 1987; 136: 657-661.

14. Todo G, Herman PG. High-resolution computed tomography of the pig lung. Invest Radiol 1986; 21 : 689-696.

15. Gehrke I, Pabst R. Cell composition and lymphocyte subsets in the bronchoalveolar lavage of normal pigs of different ages in comparison with germ-free and pneumonic pigs. Lung 1990; 168: 79-92.

16. Ichikado K, Suga M, Gushima Y, et al. Hyperoxiainduced diffuse alveolar damage in pigs: correlation between thin-section computed tomographic and pathologic findings. Radiology 2000; 216: 531-538.

17. Tamaoki J, Tagaya E, Yamawaki I, Sakai N, Nagai A, Konno K. Effect of erythromycin on endotoxininduced microvascular leakage in the rat trachea and lungs. Am J Respir Crit Care Med 1995; 151: 1582 1588.

18. Carney DE, Lutz CJ, Picone AL, et al. Matrix metalloproteinase inhibitor prevents acute lung injury after cardiopulmonary bypass. Circulation 1999; 100: 400-406.

19. Katzenstein ALA. Acute lung injury patterns: diffuse alveolar damage, acute interstitial pneumonia, bronchiolitis obliterans-organizing pneumonia. In: Katzenstein ALA, Askin FB, eds. Katzenstein and Askin's Surgical Pathology of Non-neoplastic Lung Disease, 3rd Edn. Philadelphia, W.B. Saunders, 1997; pp. 14-32.

20. Tomashefski JF Jr. Pulmonary pathology of the adult respiratory distress syndrome. Clin Chest Med 1990; 11: 593-619. 
21. Suga M, Iyonaga K, Okamoto T, et al. Characteristic elevation of matrix metalloproteinase activity in idiopathic interstitial pneumonias. Am J Respir Crit Care Med 2000; 162: 1949-1956.

22. Isobe S, Nakane PK, Broun WR. Studies on translocation of immunoglobulins across intestinal epithelium. 1. Improvements in the peroxidase-labeled antibody method for application to study of human intestinal mucosa. Acta Histochem Cytochem 1977; 10: 167-171.

23. Raghu G, Striker LJ, Hudson LD, Striker GE. Extracellular matrix in normal and fibrotic human lungs. Am Rev Respir Dis 1985; 131: 281-289.

24. Fukuda Y, Ishizaki M, Kudoh S, Kitaichi M, Yamanaka N. Localization of matrix metalloproteinases-1, -2, and -9 and tissue inhibitor of metalloproteinase- 2 in interstitial lung diseases. Lab Invest 1998; 78: 687-698.

25. Kawamura M, Yamasawa F, Ishizaka A, et al. Serum concentration of $7 \mathrm{~S}$ collagen and prognosis in patients with the adult respiratory distress syndrome. Thorax 1994; 49: 144-146.

26. Kjeldsen L, Bainton DF, Sengeløv H, Borregaard N. Structural and functional heterogeneity among peroxidase-negative granules in human neutrophils: identification of a distinct gelatinase-containing granule subset by combined immunocytochemistry and subcellular fractionation. Blood 1993; 82: 31833191.

27. Nagase H, Enghild JJ, Suzuki K, Salvesen G. Stepwise activation mechanisms of the precursor of matrix metalloproteinase 3 (stromelysin) by proteinases and (4-aminophenyl) mercuric acetate. Biochemistry 1990; 29: 5783-5789.

28. Okamoto T, Akaike T, Nagano T, et al. Activation of human neutrophil procollagenase by nitrogen dioxide and peroxynitrite: a novel mechanism for procollagenase activation involving nitric oxide. Arch Biochem Biophys 1997; 342: 261-274.

29. Galis ZS, Sukhova GK, Lark MW, Libby P. Increased expression of matrix metalloproteinases and matrix degrading activity in vulnerable regions of human atherosclerotic plaques. J Clin Invest 1994; 94: 24932503.

30. Galis ZS, Sukhova GK, Libby P. Microscopic localization of active proteases by in situ zymography: detection of matrix metalloproteinase activity in vascular tissue. FASEB J 1995; 9: 974-980. 